\title{
Acute pressure on the sciatic nerve results in rapid inhibition of the wide dynamic range neuronal response
}

Wenxue Wang ${ }^{1 \dagger}$, Wei Tan ${ }^{2+}$, Danping Luo ${ }^{2}$, Jianhua Lin², Yaoqing Yu ${ }^{3}$, Qun Wang ${ }^{2}$, Wangyeng Zhao ${ }^{2}$, Buling Wu ${ }^{2}$, Jun Chen ${ }^{3^{*}}$ and Jiman $\mathrm{He}^{2,4^{*}}$

\begin{abstract}
Background: Acute pressure on the sciatic nerve has recently been reported to provide rapid short-term relief of pain in patients with various pathologies. Wide dynamic range (WDR) neurons transmit nociceptive information from the dorsal horn to higher brain centers. In the present study, we examined the effect of a 2-min application of sciatic nerve pressure on WDR neuronal activity in anesthetized male Sprague-Dawley rats.

Results: Experiments were carried out on 41 male Sprague-Dawley albino rats weighing 160-280 grams. Dorsal horn WDR neurons were identified on the basis of characteristic responses to mechanical stimuli applied to the cutaneous receptive field. Acute pressure was applied for 2 min to the sciatic nerve using a small vascular clip. The responses of WDR neurons to three mechanical stimuli applied to the cutaneous receptive field were recorded before, and 2, 5 and 20 min after cessation of the 2-min pressure application on the sciatic nerve. Two-min pressure applied to the sciatic nerve caused rapid attenuation of the WDR response to pinching, pressure and brushing stimuli applied to the cutaneous receptive field. Maximal attenuation of the WDR response to pinching and pressure was noted 5 min after release of the 2-min pressure on the sciatic nerve. The mean firing rate decreased from $31.7 \pm 1.7 \mathrm{~Hz}$ to $13 \pm 1.4 \mathrm{~Hz}$ upon pinching $(p<0.001)$, from $31.2 \pm 2.3 \mathrm{~Hz}$ to $10.9 \pm 1.4 \mathrm{~Hz}(p<0.001)$ when pressure was applied, and from $18.9 \pm 1.2 \mathrm{~Hz}$ to $7.6 \pm 1.1 \mathrm{~Hz}(p<0.001)$ upon brushing. Thereafter, the mean firing rates gradually recovered.
\end{abstract}

Conclusions: Our results indicate that acute pressure applied to the sciatic nerve exerts a rapid inhibitory effect on the WDR response to both noxious and innocuous stimuli. Our results may partially explain the rapid analgesic effect of acute sciatic nerve pressure noted in clinical studies, and also suggest a new model for the study of pain.

Keywords: Sciatic nerve, WDR, Pain, Acute pressure

\section{Background}

Pressure stimulation of the sciatic nerve is associated with hyperalgesia [1-3]. Recently, we found that acute pressure applied to the sciatic nerve inhibited pain $[4,5]$. We then proposed that pressure causing pathologies are

\footnotetext{
* Correspondence: junchen@fimmu.edu.cn; jiman_he@brown.edu ${ }^{\dagger}$ Equal contributors

${ }^{3}$ Institute for Biomedical Sciences of Pain and Institute for Functional Brain Disorders, Tangdu Hospital, Fourth Military Medical University, Xi'an 710032, China

${ }^{2}$ Pain Medicine Program, IDD, Nanfang Hospital, Southern Medical University, Guangzhou, 510515, China

Full list of author information is available at the end of the article
}

chronic, and not acute. Long-term pressure, even at very low levels, may cause severe neural dysfunction. For example, chronic pressure applied to the sciatic nerve because of internal tension of the obturator muscle, or anatomical abnormalities in the piriformis muscle, can cause pain [6-8], and surgery to relieve pressure affords rapid relief [9-11]. On the contrary, we found that acute pressure applied to the sciatic nerve for 2 min provides rapid pain relief $[4,5,12,13]$. This relief is short-lived, with duration of minutes to hours. We also found that acute pressure on the sciatic nerve reduces clinical pain, but not experimental cold pressor pain [5]. The underlying mechanism is unknown.

\section{Biomed Central}


Spinal dorsal horn WDR neurons are the first synaptic relay point for afferent pathways and they play an important role in modifying the transmission of noxious input [14]. Injuries to sciatic nerve, such as constriction, transection, etc., can cause hyperalgesic pathologies. On the other hand, sciatic nerve block can inhibit pain. Both procedures can trigger changes in WDR activity [15-17]. For example, Sotgiu at al conducted a study in rats of the background activity of WDR neurons after sciatic nerve constriction. The WDR neurons showed high frequency discharges after ligation. The increased post-injury discharges were reduced by applying lidocaine on the peripheral site of constriction [15]. Our clinical studies showed that acute pressure block on the sciatic nerve produced rapid inhibition of pain, contrary to chronic pressure, which caused sciatica. In the present study, we examined WDR activity in rats after the application of direct acute pressure on the sciatic nerve.

\section{Methods}

\section{Animal preparations}

Experiments were carried out on 41 male Sprague-Dawley albino rats weighing 160-280 g. The animals had access to water and food ad libitum, and were maintained at a temperature of $22-26^{\circ} \mathrm{C}$ with a light/dark cycle of $12 \mathrm{~h}$. Experimental protocols were approved by the Fourth Military Medical University, People's Republic of China. The rats were anesthetized by intra-peritoneal injection with a dose of $5 \mathrm{ml} / \mathrm{kg}$ urethane-chloralose solution (containing urethane at $250 \mathrm{mg} / \mathrm{ml}$ and chloralose at $10 \mathrm{mg} / \mathrm{ml}$ ). A tracheal cannula and a left jugular vein catheter were inserted. Adequate anesthesia was confirmed intermittently during the experiment by examining the animal for spontaneous movement or whether they had an arousal response to a noxious pinch applied to the skin. The sciatic nerve was exposed high in the thigh, and was carefully isolated from the surrounding tissue. Laminectomy was performed from the T13 to L1 vertebrae to expose the lumbar enlargement for spinal neuron recording.

A pool was formed using the skin flaps surrounding the exposed sciatic nerve, and was filled with warm paraffin oil $\left(37^{\circ} \mathrm{C}\right)$ to prevent drying. Core body temperature was monitored using a thermostat probe inserted into the rectum, and was maintained at $37.5 \pm 0.5^{\circ} \mathrm{C}$ using a feedback-controlled heating pad under the ventral surface of the rat's abdomen.

\section{Application of pressure to the sciatic nerve and recording of WDR neuronal activity}

Dorsal horn WDR neurons were identified on the basis of characteristic responses to mechanical stimuli applied to the receptive field [18-20]. Extracellular single-unit recordings were made between L4 and L6 using glass capillary micro-electrodes $(10-15 \mathrm{MV}$, filled with $0.5 \mathrm{M}$ sodium acetate). The recording electrode was advanced in $2-\mu \mathrm{m}$ steps using an electronically-controlled manipulator. Light stroking and probing of the skin of the ipsilateral hind paw were used as search stimuli to identify dorsal horn neurons. WDR neurons are characterized by (1) a receptive field that consists of a small low-threshold center and a large high-threshold surround; (2) an increase in firing rate in response to brushing, application of pressure and noxious pinching of the low-threshold center; (3) a response to noxious pinching (and not to the other two stimuli) by the high-threshold surround; and (4) no evident adaptation when continuous stimulation is applied to the lowthreshold center.

After identification of a single WDR unit, a small vascular clip (pinch force of $100 \mathrm{~g}$ ) was applied to the sciatic nerve for $2 \mathrm{~min}$. The clip surfaces were lined with soft rubber, which absorb a considerable proportion of the pressure; the resulting pressure on the sciatic nerve was evaluated at approximately $30-70 \mathrm{~g}$ (We measured the pressure force of the clip on the sciatic nerves during simulation by using an elastic band. The pressure force was determined by comparing deformation of an elastic strip of rubber sandwiched in the clip with that of an elastic strip of rubber sandwiched between two flat surfaces on which a series of weights were placed). Thereafter, three types of mechanical stimuli (brushing, pressure application and pinching) were delivered to the center of the receptive field. In all rats, the responses to the three mechanical stimuli were recorded before, and 2, 5 and 20 min after release of compression. Spontaneous discharges were recorded for $10 \mathrm{~s}$ before application of any mechanical stimulus, and these were also recorded when the vascular clip was attached to the sciatic nerve and when the clip was removed until application of the first stimulus. The mean spontaneous firing rate and the response to each mechanical stimulus were analyzed. The mean firing rate during the $20 \mathrm{~s}$ period before stimulation was subtracted from each response rate. The proportionate inhibition of the response to each cutaneous stimulus was also calculated; the mean firing rate upon application of each stimulus prior to compression was taken to be $100 \%$.

The mechanical stimuli used were (1) brushing, performed by brushing of the center of the cutaneous receptive field (cRF) once per second with a hairy paint brush; (2) pressure application, performed by clamping a fold of skin between the arms of a flattened alligator clip to produce constant pressure that was not painful; and (3) noxious pinching, performed by pinching a fold of skin (with constant force) using a small serrated clip (this was painful when tested on the experimenter's skin). The force of the 
flattened clip, and the serrated clip was evaluated at approximately $100-130 \mathrm{~g}$ and $250-280 \mathrm{~g}$, respectively. For each nerve, we sequentially stimulated with brushing, pressure, and pinching for $10 \mathrm{~s}$ at each time point.

\section{Histological analysis}

A vascular clip was attached to the sciatic nerve for 2 or 20 min. Thereafter, the sciatic nerve was dissected out and biopsies were taken. The specimens were fixed in buffered $4 \%$ paraformaldehyde, washed in water, dehydrated in a graded ethanol series, cleared in xylene, embedded in paraffin, and cut into 5 - $\mu \mathrm{m}$-thick cross or longitudinal sections at the compressed sites. The longitudinal and cross sections were stained with hematoxylin and eosin (H\&E), and examined using light microscopy.

\section{Data analysis}

All results are expressed as mean $\pm \mathrm{SE}$. The data were compared using ANOVA, followed by Dunn's multiple comparisons test as post-hoc analysis. A $p$ value less than 0.05 was considered to be significant.

\section{Results}

A total of 41 WDR neurons were recorded in the L4-L6 spinal cord region, 30 in the sciatic pressure group and 11 in the sham treatment group. Figure 1 shows the mean spontaneous discharge rate at each time point (baseline, $2^{\text {nd }} \min , 5^{\text {th }} \mathrm{min}, 10^{\text {th }} \mathrm{min}$, and $20^{\text {th }} \mathrm{min}$ ). The average spontaneous discharge rate of the five time points was $1.0 \pm 0.2 \mathrm{~Hz}$ for the sciatic pressure group, and $1.1 \pm 0.3 \mathrm{~Hz}$ for the sham treatment group.

Figure 2 illustrates WDR neuronal firing caused by application of a vascular clip to the sciatic nerve, and removal of the clip. Clip attachment triggered short periods of initial firing in 26 WDR neurons, of which 18 exhibited brief continuous repetitive firing after the initial firing burst. Thereafter, the firing rate of these neurons returned to pre-attachment levels. In the remaining 4 neurons, neither initial firing nor continuous repetitive firing was observed. Removal of the vascular clip produced initial firing in 21 neurons, of which 2 exhibited continuous repetitive firing after initial transient firing. In the remaining 9 neurons, neither initial firing nor continuous firing was observed.

Figure 3 shows an example of the response of a WDR neuron to the three different stimuli (brushing, application of pressure and pinching of the skin) applied to the ipsilateral hind paw before, and 5 and 20 min after release of the 2-min pressure on the sciatic nerve. This pressure applied to the nerve caused rapid inhibition of WDR neuronal firing in response to all three types of stimulation. WDR responses to pressure and pinching recovered $20 \mathrm{~min}$ after clip removal, but recovery of the responses to brushing were slower. Some firing associated with each application of brushing at 5 minutes or 20 minutes could be due to spontaneous activity. We implemented a stimulus duration of 10 seconds to the cutaneous receptive filed. But we could not manually control the time precisely in the experiment. Consequently, there was considerable variation in the duration of stimulus application of pressure and pinching (Figure 3).

The WDR response to all applied stimuli to the cutaneous receptive filed decreased rapidly after the 2-min application of sciatic nerve pressure in our experiments (Figure 4). The greatest attenuation of the WDR response to pressure (Figure 4B) and pinching (Figure 4C) occurred $5 \mathrm{~min}$ after the release of pressure on the sciatic nerve. The mean firing rate, evoked by pressure, decreased from $31.2 \pm 2.3 \mathrm{~Hz}$ to $10.9 \pm 1.4 \mathrm{~Hz}(p<0.001)$, and that, evoked by pinching, decreased from $31.7 \pm 1.7$ $\mathrm{Hz}$ to $13 \pm 1.4 \mathrm{~Hz}(p<0.001)$. Thereafter, the WDR response gradually recovered between 5 and $20 \mathrm{~min}$. WDR responses evoked by brushing to the cutaneous receptive filed also decreased rapidly from $18.9 \pm 1.2 \mathrm{~Hz}$ to $7.6 \pm 1.1 \mathrm{~Hz}$ after application of the sciatic nerve pressure $(p<0.001)$ (Figure 4A). Thereafter, the WDR response gradually recovered, but the recovery was slower than that evoked by pressure and pinching.

To examine the intactness of the sciatic nerve after 2 minutes of pressure: we compared the histological change after applying pressure to the sciatic nerve for

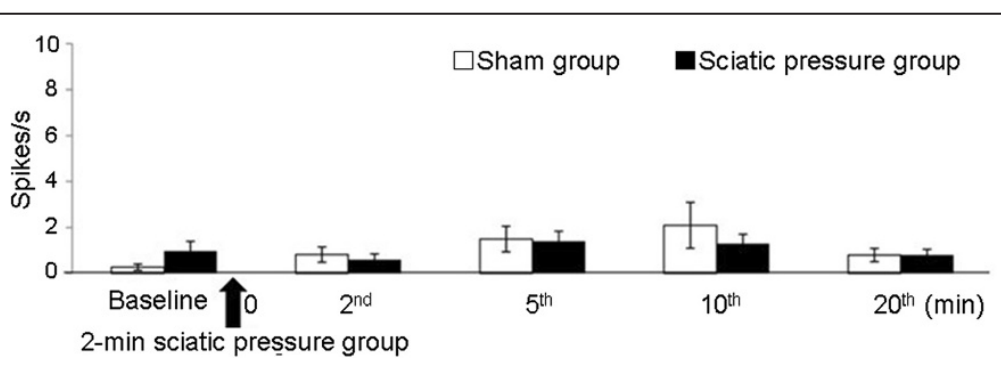

Figure 1 Spontaneous discharges for the sciatic pressure and sham groups. The time when the vascular clip was attached to the sciatic nerve is indicated by the black arrow. The "0" point represents the time at which the 2 -min period of sciatic nerve pressure was terminated, and at which the post-pressure measurements of spontaneous discharge activity commenced (measured at different time points). 


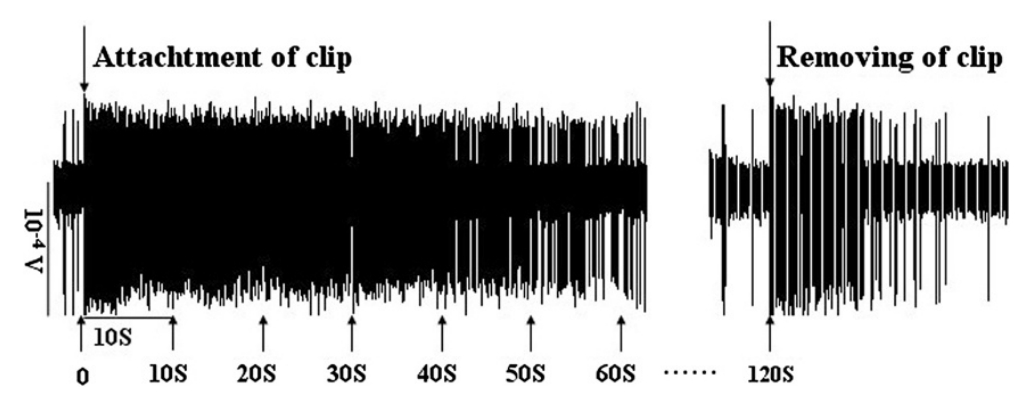

Figure 2 WDR neuron firing caused by the application of a vascular clip to, or removal of it from the sciatic nerve.

different periods $(0,2$, or 20 minutes). Longitudinal or cross sections of the compressed region of the sciatic nerve were prepared and subjected to H\&E staining. The differences between the control and the group that received pressure for 2 minutes were not significant. However, a significant difference was observed between the control and the group that received pressure for 20 minutes; the latter group showed an incurved nerve segment in longitudinal sections (Figure 5). Consistently, the cross sections showed that the nerves had narrowed in comparison to the controls. These results are in accordance with previous observations that long-term pressure has a profound effect on nerve dysfunction [21-25].

\section{Discussion}

Studies on the effects of mechanical pressure on nerves have employed different force levels (from several grams to hundreds of grams), for various time periods (from tens of minutes to several weeks), and have demonstrated that pressure duration has a profound effect on nerve function and viability [21-27]. For example, Fern and colleagues studied changes in nerve conduction caused by the deformation and ischemia induced by compression of the cat sciatic nerve [22]. These researchers recorded unitary action potentials from a dorsal root filament during stimulation of the flexor digitorum longus nerve when the sciatic nerve was subjected to $70 \mathrm{mmHg}$ of pressure. Little change was found in conduction of action potentials over the first $19 \mathrm{~min}$ of pressure. However, the second, third and fifth fastest action potentials disappeared when the duration of pressure was extended to $28 \mathrm{~min}$, and even the fastest action potential was blocked by $48 \mathrm{~min}$ of pressure [22]. These data are in accordance with clinical observations showing that prolonged pressure (even at very low levels) applied to nerves can cause severe neural dysfunction [6-11].
A

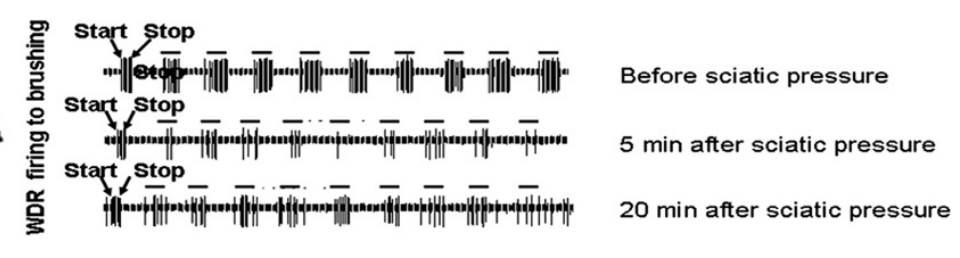

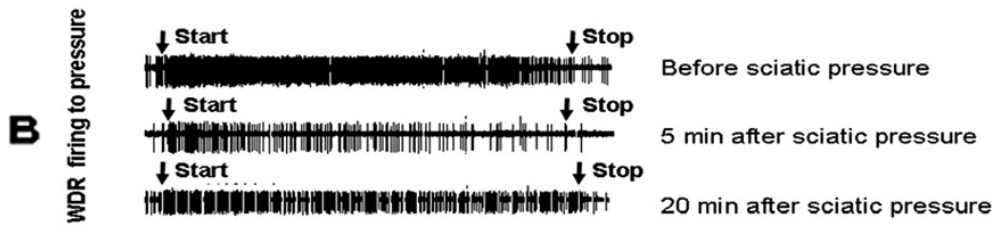

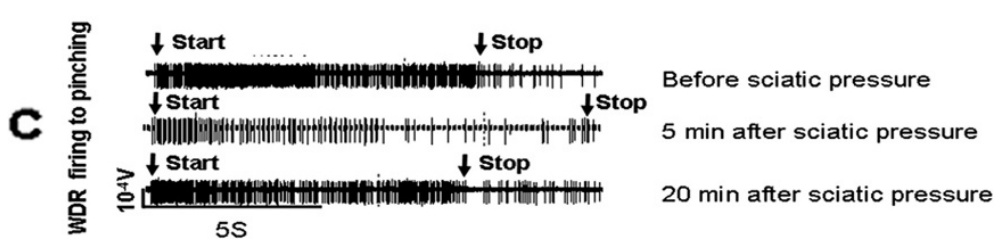

Figure 3 An example of the response of a WDR neuron to the three stimuli. The response of a WDR neuron to brushing (A), pressure (B) and pinching $(\mathbf{C})$ stimulation of the ipsilateral hind paw was recorded before, and 5 and 20 min after cessation of a 2-min application of pressure to the sciatic nerve. A horizontal bar in Figure 3A indicates firing associated with each application of brushing. 

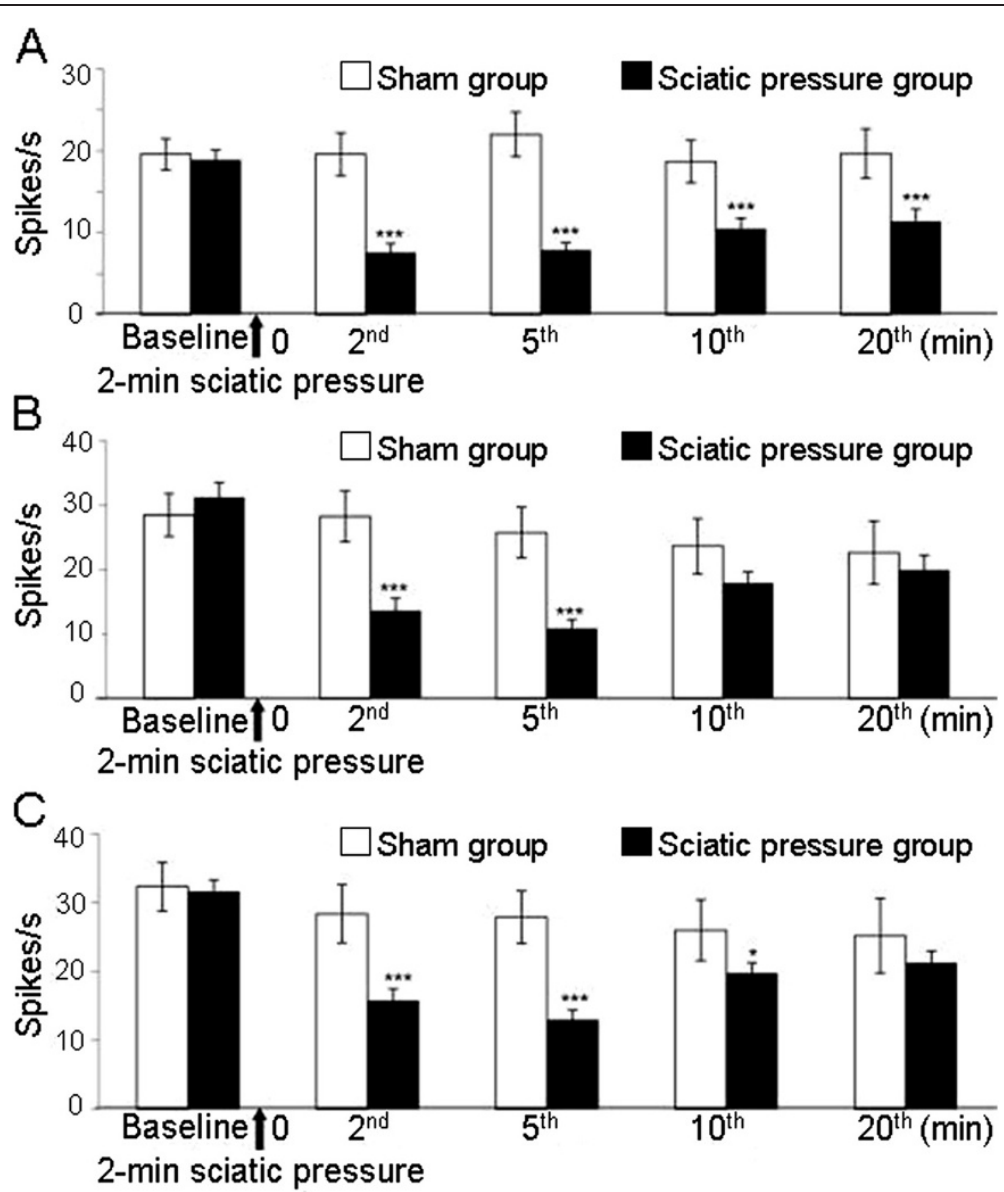

Figure 4 Time course of the mean firing rate of WDR neurons. The mean firing rate of a WDR neuron was evoked by application of brushing $(\mathbf{A})$, pressure $(\mathbf{B})$ and pinching $(\mathbf{C})$ stimuli of the skin of the ipsilateral hind paw before, and 2, 5 and 20 min after release of 2-min sciatic nerve pressure. The time that the vascular clip was attached to the sciatic nerve is indicated by the black arrow. Each point (bin) was an average of 11 nerves in the sham group, and 27 nerves in the sciatic pressure group. ${ }^{*} p<0.05,{ }^{* * *} p<0.001$, compared with the mean rate of the response before the pressure (sciatic pressure group).

In our previous clinical studies, we showed that manual pressure applied through the skin, soft tissue and muscle on the back of the leg to the sciatic nerve provides significantly more relief from pain than placebo pressure on the front of the leg. We also examined pressure on different parts of the leg, and demonstrated that effective pressure on any accessible area along the sciatic nerve will give rapid pain relief; and the effectiveness is reduced if the same manual pressure is applied at a distance from the sciatic nerve tract $[4,13]$. These previous clinical results may suggest that, in addition to activating mechanosensitive receptors in the skin and muscle afferent neurons, pressure on the sciatic nerve itself may provide significant analgesic effect. In the present study, we demonstrate that a short duration of pressure $(2 \mathrm{~min})$, directly on the sciatic nerve, causes rapid inhibition of WDR responses to both innocuous and noxious mechanical stimuli, and that the inhibitory responses recover within tens of minutes after pressure release. Thus, this animal data may partially explain the rapid analgesia in the clinical setting.

The Diffuse Noxious Inhibitory Control (DNIC) model has been frequently used for quantifying central sensitization in several pain conditions. DNIC relies on painful conditioning stimulation of one part of the body to inhibit pain in another part; the inhibition of pain is rapid and short lasting [28,29]. In our model, pressure on the sciatic nerve is stimulation from another part of the body, and the consequent inhibition induced by the stimulation is rapid and short lasting. Thus, the DNIC mechanism may explain the early inhibition caused by acute pressure on the sciatic nerve in our model.

Sensory transduction in nerves is accomplished by proteins in the membrane called ion channels, which are gated pores that allow the exchange of ions across the cell membrane. Acid-sensitive ion channels (ASIC) have been found expressed in neurons of the mammalian central and peripheral nervous systems, and proposed to 


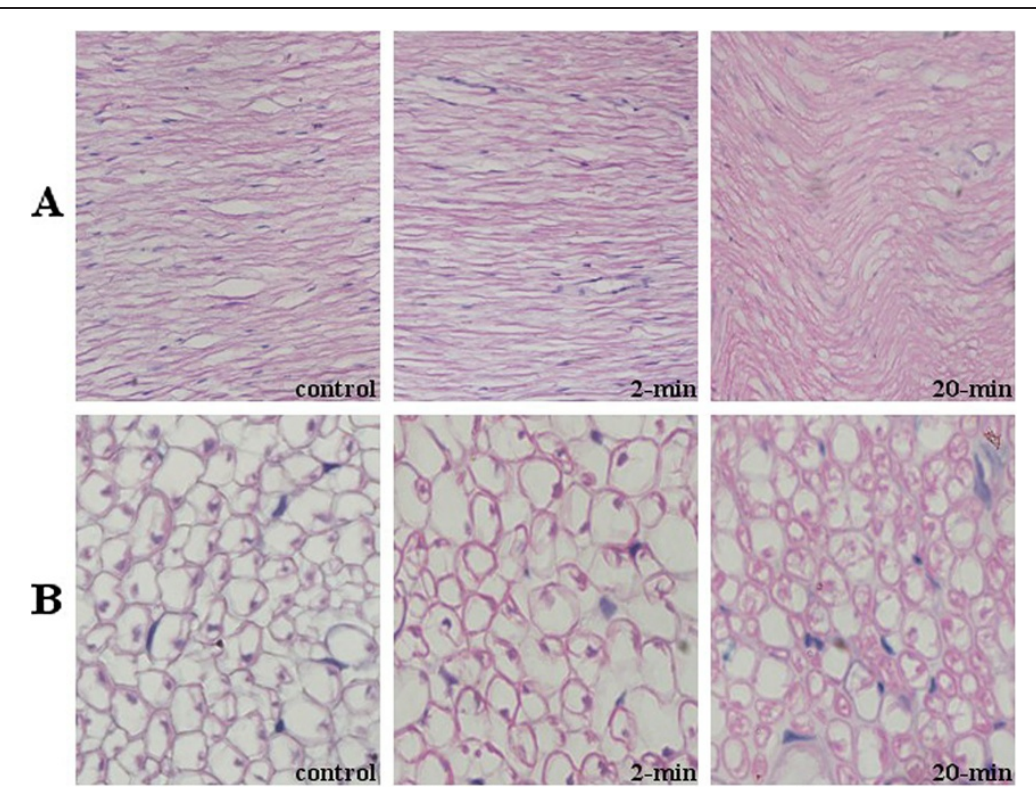

Figure $\mathbf{5}$ Intactness of sciatic nerve fibers after the pressure application. (A) longitudinal section of sciatic nerve at the compressed site (original magnification, $\times 400$ ). (B) Cross section of sciatic nerve at the compressed site (original magnification, $\times 1000$ ). 'Control': the sciatic nerve after sham treatment; '2-min': the sciatic nerve after 2 min of pressure at the site; and '20-min': the sciatic nerve after 20 min of pressure at the site.

constitute mechanoreceptors, and play an important role in responses to mechanical stimuli [30-35]. After a comparison study between ASIC1 knockout mice and wildtype mice for visceral mechanosensation, Page at al found ASIC1 contributed to visceral but not cutaneous mechanoreceptor function, and suggested that mechanosensory function in different tissues may involve different mechanisms (33). In a recent report, mice with simultaneous disruptions of ASIC1a, ASIC2, and ASIC3 genes showed increased paw withdrawal frequencies when mechanically stimulated with von Frey filaments. Moreover, in single-fiber nerve recordings of cutaneous afferents, mechanical stimulation generated enhanced activity in ASIC triple-knockouts mice compared to wild-type mice (32). Mogil et al. reported ASIC3 mice with a dominant-negative mutation were more sensitive to a number of modalities including mechanical pain, mechanical hypersensitivity after zymosan inflammation, and mechanical hypersensitivity after intramuscular injection of hypotonic saline (36). Four ASIC proteins (ASIC1, ASIC2, ASIC3, and ASIC4) have been found expressed in the sciatic nerve [36]. Thus, rapid inhibition of WDR responses to mechanical stimuli in our model may involve ASICs.

Reports on WDR responses to mechanical stimuli after application of pressure to nerves have been inconsistent. This may be attributable to differences in experimental conditions. The surfaces of the clips that compressed the sciatic nerve were covered with a soft layer of rubber in our studies, which not only absorb a considerable proportion of the pressure, but also protect the nerves from direct damage that might be caused by the clip. The actual pressure on the sciatic nerves estimated varied between 30 and $70 \mathrm{~g}$. This pressure may be several times higher than that employed in clinical studies. Hanai et al. used a clip similar to ours to compress the dorsal root or the dorsal root ganglion; the WDR responses to mechanical stimuli increased after release of the pressure [37]. These data differ from ours. The clip force in their study was $40 \mathrm{~g}$; thus, the pressure on the sciatic nerve was similar to ours; but the pressing surfaces of their clips did not have a soft rubber layer. Using clips similar to ours, Kawasaki and et al. applied much longer time pressure (30 $\mathrm{min})$, higher pressure $(120 \mathrm{~g})$, and without any soft layer on the surface of clips [38].

The WDR responses to pinch and pressure stimuli gradually recovered in both our and Kawasaki's studies after the release of the pressure on the sciatic nerve. However, in Kawasaki's study, the WDR response to an innocuous stimulus (brushing) did not show any recovery for 30 minutes after the release of the pressure on the sciatic nerve. In contrast, in our study, the WDR response to brushing, gradually recovered from 7.5 spikes/s to 11 spikes/s within 20 minutes after release of the pressure. Innocuous sensations are mainly mediated by large myelinated afferent fibers (A $\beta$ fibers), which are sensitive to pressure. Whereas, noxious sensations are mediated by fine afferent fibers (A $\delta$ and $C$ fibers) which are sensitive to oxygen deprivation $[22,39]$. Thus, the 
lack of recovery of WDR neurons to the brushing stimulus after the release of pressure on the sciatic nerve in Kawasaki's study may be attributed to damage to large myelinated afferent fibers. Gradual recovery of WDR neurons after the release of pressure in our model, which is similar to but slower than the recovery after release of pinch and pressure, may indicate that nerves were only partially injured, if at all. This is consistent with the histological data: when the injury of sciatic nerves was observed, it occurred when pressure was applied to the sciatic nerve for 20 minutes, but not when the pressure was applied for 2 minutes.

\section{Conclusions}

Acute pressure applied to the sciatic nerve exerts a rapid inhibitory effect on the WDR response to both noxious and innocuous stimuli. Our results may, in part, explain the rapid analgesic effect of acute sciatic nerve pressure noted in clinical studies, and suggest a new model for the study of pain.

\section{Competing interests}

The authors declare that they have no competing interests.

\section{Authors' contributions}

$\mathrm{JH}$ and $\mathrm{JC}$ are the primary investigators in this study. WW and $\mathrm{WT}$ were responsible for the animal experiments and drafted the manuscript. $\mathrm{DL}$, $\mathrm{L}$, YY, QW, WZ and BW helped or were responsible for part of the experiments. All authors have read and approved the final manuscript.

\section{Acknowledgements}

This work was supported partially by Talents Foundation of Nanfang Hospital (2010111501) to J.H., the National Basic Research (973) Program of China (2006CB500808), and Natural Science Foundation of China (30770668) to J.C... The authors are grateful to Dr. J. Wands (Brown University) and Dr. G. Donaldson (Buffalo) for their important help, and to all members of Institute for Biomedical Sciences of Pain, Tangdu Hospital FMMU, for their cooperation during the experiments.

\section{Author details}

'Department of Physiology, School of Life Science, Kuning 605000, China. ${ }^{2}$ Pain Medicine Program, IDD, Nanfang Hospital, Southern Medical University, Guangzhou 510515, China. Institute for Biomedical Sciences of Pain and Institute for Functional Brain Disorders, Tangdu Hospital, Fourth Military Medical University, Xi'an 710032, China. ${ }^{4}$ Rhode Island Hospital, Brown University, Providence 02903, USA.

Received: 15 February 2012 Accepted: 29 November 2012 Published: 4 December 2012

\section{References}

1. Al-Khodairy AW, Bovay P, Gobelet C: Sciatica in the female patient: anatomical considerations, aetiology and review of the literature. Eur Spine J 2007, 16:721-731

2. García S, Cofán F, Combalia A, Casas A, Campistol JM, Oppenheime F: Compression of the sciatic nerve in uremic tumor calcinosis. Neurologia 1999, 14:86-89.

3. Lin HL, Chen JT, Liu YF, Cho DY: Sciatica caused by pseudomyxoma peritonei. J Chin Med Assoc 2009, 72:39-41.

4. He J, Wu B, Zhang W, Ten G: Immediate and short-term pain relief by acute sciatic nerve press: a randomized controlled trial. BMC Anesthesiol 2007, 16:7-4.

5. He J, Jiang X, Zhao B, Xu S, Zhang F, Wei P, Chen Q: Acute pressure block of the sciatic nerve relieves clinical pain but not cold pressor pain. The Clinical Journal of Pain 2010, 26:332-338.
6. Benson ER, Schutzer SF: Posttraumatic piriformis syndrome: diagnosis and results of operative treatment. J Bone Joint Surg Am 1999, 81:941-949

7. Mullin V, de Rosayro M: Caudal steroid injection for treatment of piriformis syndrome. Anesth Analg 1990, 71:705-707.

8. Solheim LF, Siewers P, Paus B: The piriformis muscle syndrome, sciatic nerve entrapment treated with section of the piriformis muscle. Acta Orthop Scand 1981, 52:73-75.

9. Chen WS: Bipartite piriformis muscle: an unusual cause of sciatic nerve entrapment. Pain 1994, 58:269-272.

10. Meknas K, Christensen A, Johansen O: The internal obturator muscle may cause sciatic pain. Pain 2003, 104:375-380.

11. Sayson SC, Ducey JP, Maybrey JB, Wesley RL, Vermilion D: Sciatic entrapment neuropathy associated with an anomalous piriformis muscle. Pain 1994, 59:149-152.

12. He J, Wu B, Jiang $X$, Zhang F, Zhao T, Zhang W: A new analgesic method, two-minute sciatic nerve press, for immediate pain relief in man: a randomized trial. BMC Anesthesiol 2008, 25:8-1.

13. He J, Chen Q: General responses to questions regarding acute sciatic nerve pressure for pain relief. Pain Medicine 2010, 11:1139-1140.

14. Staud R: Evidence of involvement of central neural mechanisms in generating fibromyalgia pain. Curr Rheumatol Rep. 2002, 4:299-305.

15. Sotgiu ML, Biella G, Riva L: A study of early ongoing activity in dorsal horn units following sciatic nerve constriction. Neuroreport 1994, 5:2609-2612.

16. Sotgiu ML, Biella G, Riva L: Poststimulus afterdischarges of spinal WDR and NS units in rats with chronic nerve constriction. Neuroreport 1995, 6:1021-1024.

17. Sotgiu ML, Biella G: Contribution of central sensitization to the pain-related abnormal activity in neuropathic rats. Somatosens Mot Res 2000, 17:32-38.

18. You HJ, Dahl Morch C, Chen J, Arendt-Nielsen L: Simultaneous recordings of wind-up of paired spinal dorsal horn nociceptive neuron and nociceptive flexion reflex in rats. Brain Res 2003, 960:235-245.

19. Zheng JH, Chen J, Arendt-Nielsen L: Complexity of tissue injury-induced nociceptive discharge of dorsal horn wide dynamic range neurons in the rat, correlation with the effect of systemic morphine. Brain Res 2004 1001:143-149.

20. Zheng JH, Jian Z, Chen J: Detection of deterministic behavior within the tissue injury-induced persistent firing of nociceptive neurons in the dorsal horn of the rat spinal cord. J Comput Neurosci 2002, 13:23-34.

21. Dahlin LB, Shyu BC, Danielsen N, Andersson SA: Effects of nerve compression or ischaemia on conduction properties of myelinated and non-myelinated nerve fibres, An experimental study in the rabbit common peroneal nerve. Acta Physiol Scand 1989, 136:97-105.

22. Fern R, Harrison PJ: The contribution of ischaemia and deformation to the conduction block generated by compression of the cat sciatic nerve. Exp Physiol 1994, 79:583-592.

23. Gelberman RH, Szabo RM, Williamson RV, Dimick MP: Sensibility testing in peripheral-nerve compression syndromes. An experimental study in humans. J Bone Joint Surg Am 1983, 65:632-638.

24. Lundborg G, Dahlin LB: The pathophysiology of nerve compression. Hand Clin. 1992, 8:215-227.

25. Macgregor RJ, Sharpless SK, Luttges MW: A pressure vessel model for nerve compression. J Neurol Sci 1975, 24:299-304.

26. Ochoa J, Fowler TJ, Gilliatt RW: Anatomical changes in peripheral nerves compressed by a pneumatic tourniquet. J Anat 1972, 113:433-455.

27. Powell HC, Myers RR: Pathology of experimental nerve compression. Lab Invest 1986, 55:91-100.

28. Le Bars D, Dickenson AH, Besson JM: Diffuse nosious inhibitory control (DNIC). I. Effects on dorsal horn convergent neurons in the rat. Pain 1979, 6:283-304.

29. Le Bars D, Dickenson AH, Besson JM: Diffuse nosious inhibitory control (DNIC). II. Lack of effect on non-convergent neurons, supraspinal involvement and theoretical implications. Pain 1979, 6:305-327.

30. Geffeney SL, Goodman MB: How we feel: ion channel partnerships that detect mechanical inputs and give rise to touch and pain perception. Neuron 2012, 74:609-619.

31. Kang S, Jang JH, Price MP, Gautam M, Benson CJ, Gong H, Welsh MJ, Brennan TJ: Simultaneous disruption of mouse ASIC1a, ASIC2 and ASIC3 genes enhances cutaneous mechanosensitivity. PLoS One 2012, 7:e35225. 
32. Page AJ, et al: The ion channel ASIC1 contributes to visceral but not cutaneous mechanoreceptor function. Gastroenterology 2004, 127:1739-1747.

33. Page AJ, Brierley SM, Martin CM, Hughes PA, Blackshaw LA: Acid sensing ion channels 2 and 3 are required for inhibiton of visceral nociceptors by benzamil. Pain 2007, 133:150-160.

34. Price MP, Thompson RJ, Eshcol JO, Wemmie JA, Benson CJ: Stomatin Modulates Gating of Acid-sensing Ion Channels. J Biol Chem 2004, 279:53886-53891.

35. Mogil JS, Breese NM, et al: Transgenic expression of a dominant-negative ASIC3 subunit leads to increased sensitivity to mechanical and inflammatory stimuli. J Neurosci 2005, 25:9893-9901.

36. Alvarez dela Rosa D, Zhang P, Shao D, White F, Canessa CM: Functional implications of the localization and activity of acid-sensitive channels in rat peripheral nervous system. Proc Natl Acad Sci USA 2002, 99:326-331.

37. Hanai $F$, Matsui $N$, Hongo $N$ : Changes in responses of wide dynamic range neurons in the spinal dorsal horn after dorsal root or dorsal root ganglion compression. Spine 1996, 21:1408-1415.

38. Kawasaki M, Ushida T, Tani T, Yamamoto H: Changes of wide dynamic range neuronal responses to mechanical cutaneous stimuli following acute compression of the rat sciatic nerve. J Orthop Sci 2002, 7:111-116.

39. Light AR, Perl ER: Peripheral sensory systems. In Periperal neuropathy. Edited by Dyck PJ, Thomas PK. Philadelphia: WB Saunders; 1993:149-163.

doi:10.1186/1471-2202-13-147

Cite this article as: Wang et al:: Acute pressure on the sciatic nerve results in rapid inhibition of the wide dynamic range neuronal response. BMC Neuroscience 2012 13:147.

\section{Submit your next manuscript to BioMed Central and take full advantage of:}

- Convenient online submission

- Thorough peer review

- No space constraints or color figure charges

- Immediate publication on acceptance

- Inclusion in PubMed, CAS, Scopus and Google Scholar

- Research which is freely available for redistribution 